\title{
Refractometer probe based on reflective carbon nanotube-modified microfiber Bragg grating
}

\author{
Biqiang Jiang, ${ }^{1,2,}{ }^{*}$ Meng Xue, ${ }^{1}$ Chenyang ZhaO,${ }^{1}$ Dong MaO, ${ }^{1}$ Kaiming \\ ZHOU, ${ }^{2,3}$ LIN ZHANG, ${ }^{2}$ AND JIANLIN ZHAO, ${ }^{1}$
}

\author{
${ }^{1}$ Key Laboratory of Space Applied Physics and Chemistry, Ministry of Education, and the Shaanxi Key Laboratory of Optical Information \\ Technology, School of Science, Northwestern Polytechnical University, Xi'an 710072, China \\ ${ }^{2}$ Aston Institute of Photonic Technologies, Aston University, Birmingham B4 7ET, U.K. \\ ${ }^{3}$ State Key Laboratory of Transient Optics and Photonics, Xi'an Institute of Optics and Precision Mechanics, Chinese Academy of Sciences, Xi'an \\ 710119, China \\ *Corresponding author: bqjiang@nwpu.edu.cn
}

Received XX Month XXXX; revised XX Month, XXXX; accepted XX Month XXXX; posted XX Month XXXX (Doc. ID XXXXX); published XX Month XXXX

A carbon nanotube (CNT)-modified microfiber Bragg grating (MFBG) is proposed to measure the refractive index with strong enhancement of the sensitivity in low refractive index region. The introduction of the CNT layer influences evanescent field of the MFBG and causes modification of the reflection spectrum. With the increase of the surrounding refractive index (SRI), we observe significant attenuation to the peak of the Bragg resonance while its wavelength almost remains unchanged. Our detailed experimental results disclose that the CNT-MFBG demonstrates strong sensitivity in the low refractive index range of 1.333 1.435, with a peak intensity up to -53.4 $\mathrm{dBm} / \mathrm{RIU}$, which is 15-fold higher than that of the uncoated MFBG. Therefore, taking advantage of the CNT-induced evanescent field enhancement, the reflective MFBG probe presents strong sensing capability in biochemical fields. (C) 2016 Optical Society of America

OCIS codes: (060.2340) Fiber optics components; (060.3735) Fiber Bragg gratings; (060.2370) Fiber optics sensors.

http://dx.doi.org/10.1364/AO.99.099999

\section{INTRODUCTION}

Fiber Bragg grating (FBG), a periodic perturbation of the refractive index along the fiber core, has emerged as one of the most useful optical fiber devices for thermal and mechanical sensing thank to the advantage of wavelength encoding [1]. However, due to confinement of optical mode by the thick cladding, FBGs fabricated in conventional single-mode optical fiber cannot be used directly for sensing of surrounding medium's refractive index (SRI) [2]. To get the guided mode in the fiber core out of the cladding and make light to interact with surroundings, etching or polishing of the fiber cladding is an effective approach [3-5]. The diameter, uniformity and length of the etched fiber can manipulate the intensity of evanescent field, leading to the change of the reflectivity of the grating and thus its SRI sensitivity [6-8]. Moreover, FBGs were also directly written in microstructured optical fiber (MOF) without etching the cladding to improve their SRI sensing performance for specific applications, which is based on the intrinsic interaction between the guided mode and medium introduced into the holes of MOF $[9,10]$. Nevertheless, the devices made in such a way suffer from rather limited detection dynamic range and low sensitivity, especially for some biological or environmental applications which involve aqueous solutions with low level of SRI [5, 11]. Thus alternative approaches for enhancing SRI sensitivity constantly pursued. As such, coating of graphene (oxide) or carbon nanotubes (CNTs) on the etched FBG surface to redistribute the evanescent field were used for gas or bio-sensing with high sensitivity [12-14].

With their remarkable optical properties and merits of good stability, ease of fabrication, as well as high compatibility to silica fibers, CNTs have been applied to many electro-optical devices [15-17]. Also, as excellent materials for surface modification of optical fiber, CNTs have been integrated with various fiber structures, including photonic crystal fiber, tilted FBG (TFBG), and long-period fiber grating (LPG) [18-22]. By exploiting intervention of CNTs with the evanescent field of the above devices, great improvements to refractive index responsivity were demonstrated for chemical and biological parameter measurements.

In this paper, we report the experimental demonstration on a refractometer probe based on CNT-coated micro-FBG (CNT-MFBG). Deposited on the surface of the etched FBG and staying in the mode field of the fiber which spreads across the thinned cladding, the CNT layer redistributes the evanescent field of the MFBG effectively due to light-matter interaction. Also, the variation of SRI can change the effective index of CNT layer, leading to MFBG's spectral evolution accordingly. By analyzing peak intensity, we show the enhancement of the refractometric sensitivity in the desirable low refractive index 
range of 1.333 1.435. Compared with the aforereported LPG and TFBG, the reflective MFBG sensing probe is of easier fabrication, potential low cost detection and suitable for a broad range of applications for the remote monitoring of a variety of biochemical parameters.

\section{PRINCIPLES, EXPERIMENTAL SETUP AND SENSING PROBE FABRICATION}

In conventional single-mode fiber FBGs, according to the coupling mode theory and phase matching condition, the guided mode is reflected back due to the strong coupling between the forward and backward propagating core modes at Bragg wavelength $\lambda_{\mathrm{B}}$, given by

$$
\lambda_{\mathrm{B}}=2 n_{\mathrm{eff}} \Lambda \text {. }
$$

Where $n_{\text {eff }}$ is the effective modal index, and $\Lambda$ is the period of grating. The Bragg wavelength depends on any perturbation imposed on the fiber that changes one of the parameters of Eq. (1). At the same time, the full width half maximum (FWHM) bandwidth $\Delta \lambda$ of the grating can be determined by $n_{\text {eff, }}$ the modulation depth $\Delta n$ of the refractive index and the number $N$ of grating planes, in a relation given by [23]

$$
\Delta \lambda=\lambda_{\mathrm{B}} \sqrt{\left(\frac{\Delta n}{n_{\mathrm{eff}}}\right)^{2}+\left(\frac{1}{N}\right)^{2}} .
$$

From Eqs. (1) and (2), in a conventional FBG, the parameters of $n_{\text {eff }}$ and $\Lambda$ are independent of the refractive index of the surrounding medium due to the thick cladding, so the Bragg wavelength and spectral bandwidth remains constant with the SRI change. However, the evanescent wave of guided mode of MFBGs can leak into surrounding material, making them sensitive to SRI. The effective refractive index of a MFBG can be affected by its diameter, and has a nonlinear dependence on the SRI [5, 7]. Generally, smaller MFBG will have a shorter Bragg wavelength, broader spectrum and higher sensitivity to SRI [24, 25].

CNTs, as one of the most attractive nanofunctional materials for optical fiber surface modification, can effectively change the evanescent field distribution around the surface of an MFBG. Having high refractive index (real part), the thin CNT layer coated around the MFBG surface will raise the effective index of the guided mode, leading to its reflected wavelength red-shifting. Also, with increasing of SRI, the mode field is less confined in the core region but evanescent wave will be enhanced, endowing a stronger interaction with the surrounding medium. However, the imaginary part of the CNT refractive index induces absorption and leads to a variation of the MFBG's reflection power with the change of the ambient environment [8].

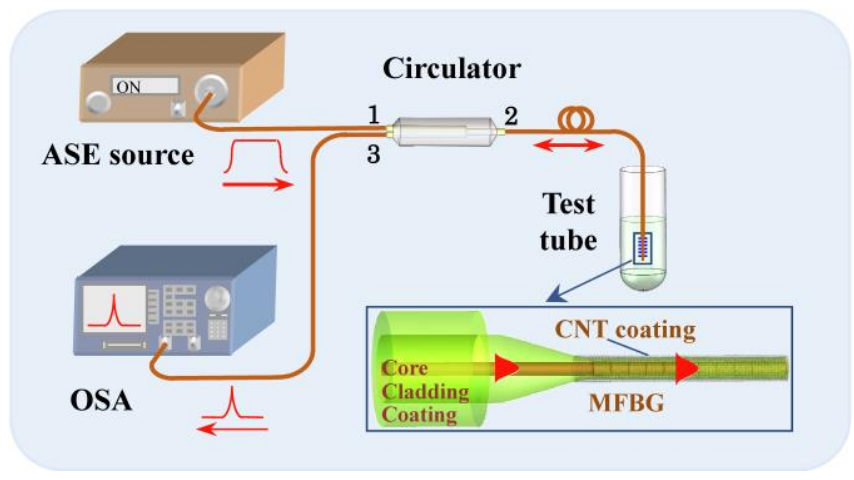

Fig. 1. Experimental setup for refractive index measurement; the inset shows the schematic diagram of MFBG with CNT coating.
Figure 1 depicts the experimental setup for reflective index measurement. Launched from an amplified spontaneous emission (ASE) source, the light is reflected back from the sensing probe via a fiber-optic circulator and monitored using an optical spectrum analyzer (OSA) with minimum wavelength resolution of $0.02 \mathrm{~nm}$. The prepared solutions of glycerol with different concentrations in the test tube are employed to examine the sensing performance of the uncoated MFBG and CNT-MFBG. The proposed CNT-MFBG structural model is shown in the inset of Fig. 1, where the CNTs are deposited around the grating surface.

In the experiment, the MFBG was etched uniformly from a standard FBG with a grating length of $\sim 5 \mathrm{~mm}$ by using diluted hydrofluoric acid. Since the diameter of the microfiber plays an important role to its effective index and evanescent field intensity of the guided mode, the SRI sensitivity will change accordingly $[7,26]$. So, in order to ensure appropriate reflectivity of the MFBG and relative robust structure, the etched MFBG diameter was reduced to $\sim 13.12 \mu \mathrm{m}$ when the MFBG just started to show spectral attenuation, implying the evanescent wave begun to interact with the liquid. The employed single-wall CNTs were synthesized through catalytic chemical vapor deposition method $[15,20]$. By using of an ultrasonic cleaner, the CNTs were dispersed in deionized (DI) water with sodium dodecyl benzene sulfonate (SDBS) to form $0.5 \mathrm{mg} / \mathrm{ml}$ CNT solution. Furtherly, the CNT dispersion was centrifuged at $12000 \mathrm{~g}$ for several hours to prevent the agglomeration. After the centrifugation, the supernatant was collected. Following the evanescent light-induced CNT deposition method [27], the prepared MFBG was immersed in the CNT dispersion to form a dense CNT layer on the grating surface. During the deposition, the broadband light with the power of $20 \mathrm{~mW}$ was input into the MFBG, and the spectral evolution was monitored by the OSA. Afterwards, the MFBG was rinsed in DI water for several times to remove the floating CNTs and to test the stability. Figures 2(a) and 2(b) display the microscope image and SEM (scanning electron microscopy) image of the fabricated device, respectively, proving the CNTs tightly deposited around the MFBG surface.

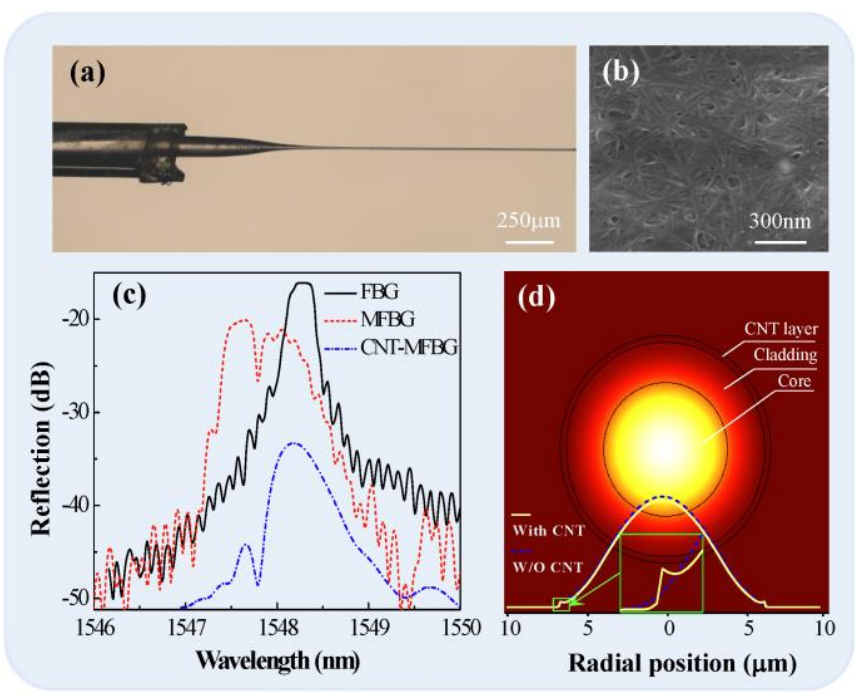

Fig. 2. (a) Optical microscope image of the integrated CNT-MFBG; (b) SEM image of CNT-MFBG surface; (c) measured reflection spectra of the initial FBG, etched MFBG, and CNT-MFBG;(d) modes and radial distributions of the simulated guided mode in MFBG with and without CNT coating at $1550 \mathrm{~nm}$ with a zoomed profile at the fiber boundary.

During the whole fabrication process, the reflection spectra of the original FBG, the etched MFBG before and after CNT deposition were 
recorded by the OSA. As depicted in Fig. 2(c), after etching, the FBG's reflection peak blue-shifts by $0.62 \mathrm{~nm}$ due to the effective index change caused by the reduced cladding. Meanwhile, the reflection power was slightly reduced and small spectrum splitting occurred, as shown in Fig. 2(c). This may be explained by the mismatch of the numerical aperture between the unetched and etched fiber regions depending on the refractive indices of core, cladding and the surrounding medium [6, 7] and the small defect of surface introduced by the etching. After the CNT deposition, the MFBG's reflection peak red-shifted by $0.51 \mathrm{~nm}$ due to the increase of the effective index and returned back to a smoother spectrum due to the decrease of modal interference, as shown in Fig.2(c), and the intensity significantly decreased caused by the interaction between the evanescent field and the CNT layer. In order to understand the CNT layer's contribution to the light field, the mode field profile of the MFBG with and without CNT layer at a wavelength of $1550 \mathrm{~nm}$ was numerically analyzed using the finite element method (FEM), and the results are shown in Fig. 2(d). From the figure, we clearly see an abrupt enhancement of the light field at the fiber boundary (zoomed in the inset of Fig. 2(d)), caused by the CNT layer's high refractive index which can be affected by the surroundings. However, the mode field intensity in the thick microfiber core have a slight decrease. Therefore, according to aforementioned principles, we can detect the SRI variation by monitoring the change in both wavelength and intensity of CNT-MFBG's Bragg resonance.

\section{EXPERIMENTAL RESULTS AND DISCUSSIONS}

To measure refractometric responses of the MFBG and CNT-MFBG, we prepared various glycerol solutions with refractive indices ranging from 1.333 to 1.451 . These solutions were injected into the test tube in turn, and the MFBG probe was inserted in the solution tube, and then the corresponding reflection spectra were recorded using an OSA. After each solution measurement, the MFBG probe was rinsed with distilled water and ethanol several times to remove the residual glycerol solution, and we could observe the spectrum reverting to the original one in air. During the experiment, the temperature was kept constant at $20^{\circ} \mathrm{C}$ to avoid any uncertainty caused by the temperature fluctuation.

We firstly examined the refractometric response of the MFBG without CNT coating. Figure 3(a) displays the evolution of the MFBG reflection spectra measured in solutions of a series of refractive indices, which are $1.333,1.358,1.384,1.413,1.435$, and 1.451, respectively. Obvious spectral change can be observed, showing the central wavelength shifting towards a longer wavelength and the spectral narrowing because of the increase of the effective index caused by an increasing SRI, but with only slight peak attenuation. Variations of spectral characteristics including the Bragg wavelength and a $6 \mathrm{~dB}$ bandwidth for covering the whole spectrum are analyzed, as depicted in Fig. 3(b). The wavelength and the bandwidth exhibit exponentially rise and fall with the increasing of SRI, respectively, and in the desirable low refractive index region of 1.33 1.40, the MFBG gives a lower SRI sensitivity, similar to that of normal LPG and other fiber-based refractive index sensing structure [3, 28-30].

Then, with the same method, the refractometric response of the CNT-MFBG probe was evaluated, and the results are shown in Fig. 4. From the spectral variations in Fig. 4(a), we observe that the total reflection power shows a significant decrease due to the change of the extinction ratio of the evanescent wave of the guided mode in the fiber boundary and the absorption effect of CNT coating [19], while the wavelength kept almost constant with variation of SRI. This interesting change trend is caused by the complex value of CNT layer refractive index, which is higher than that of the fiber cladding. The thin layer of the high index would redistribute the evanescent wave, making it stronger in the coating in such an inverted refractive index profile in the fiber radial direction, but also fix the resonant wavelength of the grating, similar to that of LPG surrounded by the solution with a higher refractive index [30]. However, the absorption property of CNT layer can be influenced by surrounding medium, leading to orderly attenuation of the reflection peak, as shown in Fig. 4(a). More interestingly, as depicted in Fig. 4(b), the bandwidth is slightly decreasing in a linear manner with an increasing SRI in the investigated SRI range of 1.333 1.435, and the SRI sensitivity estimated is $\sim-1.229 \mathrm{~nm} / \mathrm{RIU}$ (RIU stands for "refractive index unit"). Meanwhile, the peak wavelength only fluctuates by $\sim 0.072 \mathrm{~nm}$.

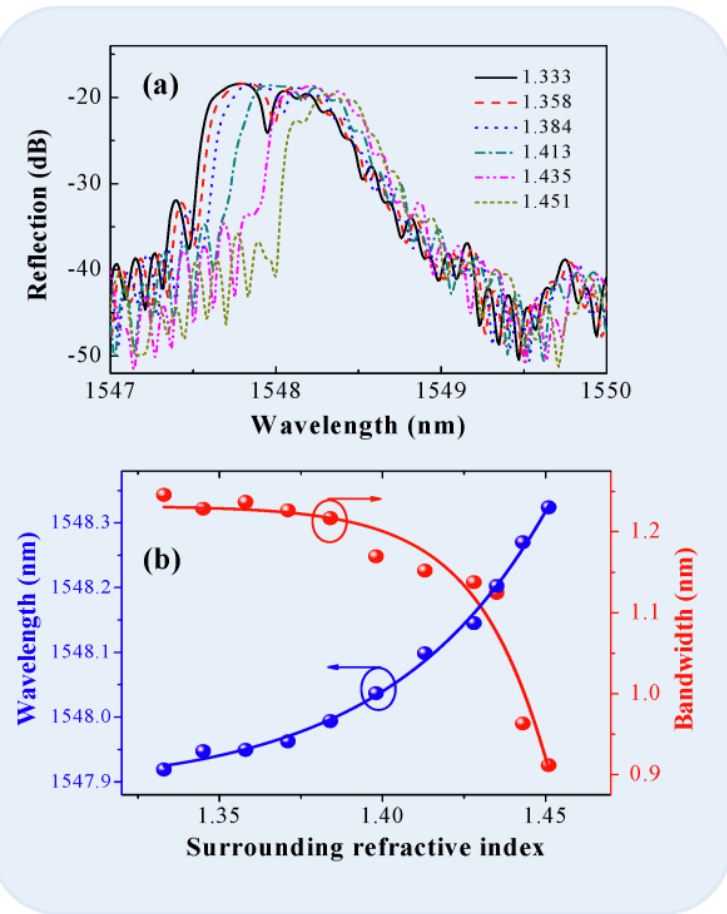

Fig. 3. (a) Reflection spectra of the MFBG immersed in the solutions with different refractive indices; (b) Bragg wavelength (blue) and bandwidth (red) variations of the MFBG versus the SRI.

Furthermore, to clearly demonstrate the effect of the CNT coating on the reflection power change of Bragg resonance, we analyzed the peak attenuation with the SRI, as shown in Fig. 5 . It can be clearly seen that in the SRI ranges from 1.333 to 1.443 , the peak intensity of the bare MFBG only slightly decreases, while that of the MFBG with CNT coating has a clear downward trend, which shows properties similar to our previous work [20]. Also, the peak intensity decreases linearly with the SRI, and in the range of 1.333 1.435, the response sensitivity is up to $53.4 \mathrm{dBm} / \mathrm{RIU}$ (with an $R$-square value 0.996 of the linear fitting curve) from $-3.46 \mathrm{dBm} / \mathrm{RIU}$ of the uncoated MFBG, corresponding to an enhancement of more than 15 -fold. The maximum refractive index error of $\sim 0.008$ can be obtained from Fig. 5. Compared to the bare MFBG, due to the presence of CNT coating, the peak intensity of the CNT-MFBG probe exhibits a linear change with the SRI, and the sensitivity is greatly enhanced in the low refractive index of 1.333 1.435. Also, when the SRI is more than 1.435, the spectral properties, including the bandwidth and peak intensity, will remain unchanged. In addition, when the CNT-MFBG is placed in air or the same solution at different times, the probe shows excellent repeatability, evidenced by the same spectral shift and attenuation. Therefore, by exploiting CNT coating with the complex refractive index and absorption properties related to the surroundings, both the 
bandwidth and peak intensity change accordingly, while the resonant wavelength remains the same. Thus such a refractometer probe may be detected by low-cost power measurement using a photodiode detector or power meter. It must be emphasized that this reflective sensing probe can provide a new sensitive window for biochemical sensing field since its sensing range can cover the desired lower refractive index region in most aqueous solutions.

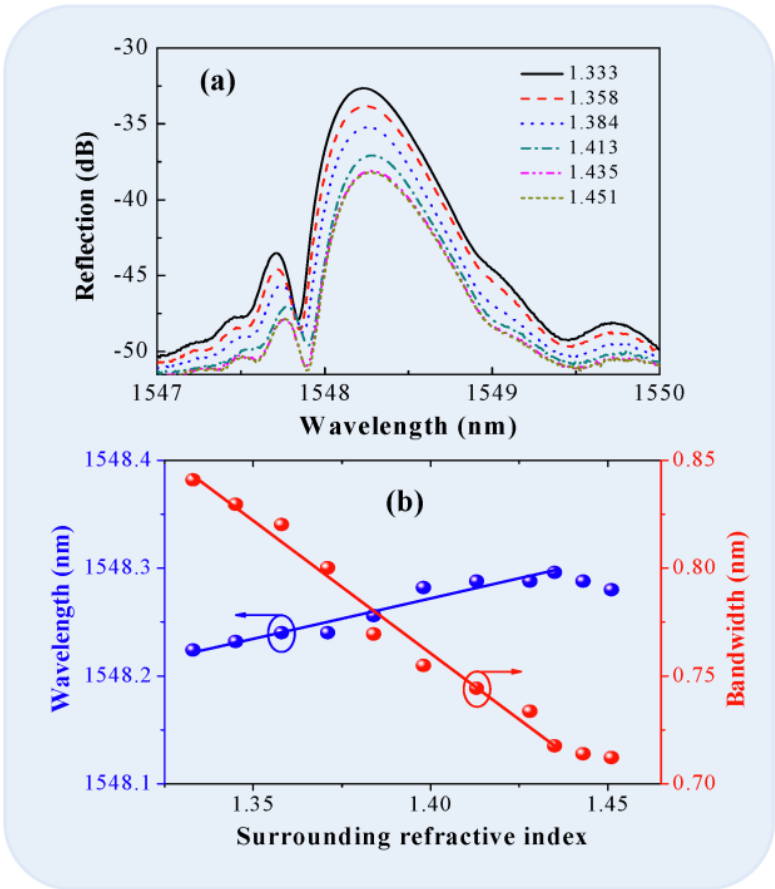

Fig. 4. (a) Reflection spectra of the CNT-MFBG immersed in the solutions with different refractive indices; (b) Bragg wavelength (blue) and bandwidth (red) variations of the CNT-MFBG versus the SRI.

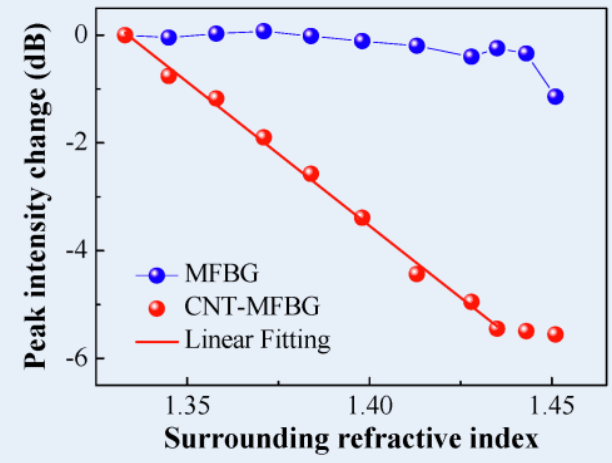

Fig. 5. Peak intensity changes of MFBG (blue) and CNT-MFBG (red) versus the SRI.

\section{CONCLUSIONS}

A reflective refractometer probe by depositing CNTs on an etched MFBG has been experimentally demonstrated. By integrating the CNT layer with a complex refractive index around the MFBG, the evanescent field in the fiber boundary can be enhanced and modulated. Compared with the original FBG and etched MFBG, the fabricated CNTMFBG modifies the response trend of the spectral properties. As a result, the peak intensity of Bragg resonance linearly decreases with the SRI, and the sensitivity in the desired refractive index region of 1.333 1.435 is greatly improved. Moreover, with the SRI increase, only the peak intensity changes, while the Bragg wavelength almost remains constant, which may provide a feasibility to achieve the simultaneous measurements of the SRI and the ambient temperature. Also, the sensing performance could be further optimized by modifying the surface properties of CNTs with chemical or physical functionalization methods. In addition, the demonstrated CNT-MFBG may be interrogated using low-cost power based measurements. Therefore, the proposed CNT-modified MFBG probe could provide a new alternative sensing technique for environmental and human health monitoring as well as other biochemical sensing applications.

Funding. National Natural Science Foundation of China (NSFC) (61505165, 61405161, 11404263); Natural Science Basic Research Plan in Shaanxi Province of China (2016JQ6032); Marie SkłodowskaCurie Individual Fellowships in the European Union's Horizon 2020 Research and Innovation Programme (660648).

\section{REFERENCES}

1. K. O. Hill, and G. Meltz, "Fiber Bragg grating technology fundamentals and overview," J. Lightw. Technol. 15, 1263-1276 (1997).

2. J. Albert, "Tilted fiber Bragg gratings as multi-sensors," Opt. Photon. News 22, 28-33 (2011).

3. K. Zhou, X. Chen, L. Zhang, and I. Bennion, "High-sensitivity optical chemsensor based on etched D-fibre Bragg gratings," Electron. Lett. 40, 232-234 (2004).

4. L. Sang-Mae, S. S. Saini, and J. Myung-Yung, "Simultaneous measurement of refractive index, temperature, and strain using etched-core fiber Bragg grating sensors," IEEE Photon. Technol. Lett. 22, 1431-1433 (2010).

5. A. N. Chryssis, S. M. Lee, S. B. Lee, S. S. Saini, and M. Dagenais, "High sensitivity evanescent field fiber Bragg grating sensor," IEEE Photon. Technol. Lett. 17, 1253-1255 (2005).

6. A. Cusano, D. Paladino, A. C. A. ladicicco, and S. Campopiano, "Fiber Bragg grating evanescent wave sensors for chemical and biological applications," in Fiber Bragg Grating Sensors: Recent Advancements, Industrial Applications and Market Exploitation(Bentham Science, 2011), pp. 238-269.

7. A. Iadicicco, A. Cusano, S. Campopiano, A. Cutolo, and M. Giordano, "Thinned fiber Bragg gratings as refractive index sensors," IEEE Sensors Journal 5, 1288-1295 (2005).

8. B. Yao, Y. Wu, L. Jia, Y. Rao, Y. Gong, and C. Jiang, "Mode field distribution of optical transmission along microfiber affected by CNT films with complex refraction index," J. Opt. Soc. Am. B 29, 891-895 (2012).

9. A. Cusano, D. Paladino, and A. ladicicco, "Microstructured fiber Bragg gratings," J. Lightwave Technol. 27, 1663-1697 (2009).

10. X. Fang, C. R. Liao, and D. N. Wang, "Femtosecond laser fabricated fiber Bragg grating in microfiber for refractive index sensing," Opt. Lett. 35, 1007-1009 (2010).

11. T. Guo, F. Liu, B.-O. Guan, and J. Albert, "[INVITED] Tilted fiber grating mechanical and biochemical sensors," Opt. Laser Technol. 78, Part B, 19-33 (2016).

12. Y. Wu, B. Yao, A. Zhang, Y. Rao, Z. Wang, Y. Cheng, Y. Gong, W. Zhang, Y. Chen, and K. S. Chiang, "Graphene-coated microfiber Bragg grating for high-sensitivity gas sensing," Opt. Lett. 39, 1235-1237 (2014).

13. B. C. Yao, Y. Wu, D. J. Webb, J. H. Zhou, Y. J. Rao, A. Pospori, C. B. Yu, Y. Gong, Y. F. Chen, and Z. G. Wang, "Graphene-based D-shaped polymer FBG for highly sensitive erythrocyte detection," IEEE Photon. Technol. Lett. 27, 2399-2402 (2015).

14. Sridevi S, K. S. Vasu, N. Jayaraman, S. Asokan, and A. K. Sood, "Optical bio-sensing devices based on etched fiber Bragg gratings coated with 
carbon nanotubes and graphene oxide along with a specific dendrimer," Sens. Actuators B Chem. 195, 150-155 (2014).

15. D. Mao, B. Jiang, W. Zhang, and J. Zhao, "Pulse-state switchable fiber laser mode-locked by carbon nanotubes," IEEE Photon. Technol. Lett. 27, 253-256 (2015).

16. Y. C. Chen, N. R. Raravikar, L. S. Schadler, P. M. Ajayan, Y. P. Zhao, T. M. Lu, G. C. Wang, and X. C. Zhang, "Ultrafast optical switching properties of single-wall carbon nanotube polymer composites at $1.55 \mu \mathrm{m}$," Appl. Phys. Lett. 81, 975-977 (2002).

17. B. Xu, M. Omura, M. Takiguchi, A. Martinez, T. Ishigure, S. Yamashita, and T. Kuga, "Carbon nanotube/polymer composite coated tapered fiber for four wave mixing based wavelength conversion," Opt. Express 21, 3651-3657 (2013).

18. L. Jia, Y. Wu, B. Yao, F. Yang, and Y. Rao, "A sensitivity enhanced 359gas sensor based on carbon nanotubes around microfiber," Proc. 360SPIE 8351,835120 (2012).

19. Y. C. Tan, Z. Q. Tou, V. Mamidala, K. K. Chow, and C. C. Chan, "Continuous refractive index sensing based on carbon-nanotubedeposited photonic crystal fibers," Sens. Actuators B Chem. 202, 10971102 (2014).

20. B. Jiang, X. Lu, D. Mao, Y. Wang, W. Zhang, X. Gan, and J. Zhao, "Carbon nanotube-deposited tilted fiber Bragg grating for refractive index and temperature sensing," IEEE Photon. Technol. Lett. 28, 994-997 (2016).

21. G. E. Villanueva, M. B. Jakubinek, B. Simard, C. J. Oton, J. Matres, L.-Y. Shao, P. Pérez-Millán, and J. Albert, "Linear and nonlinear optical properties of carbon nanotube-coated single-mode optical fiber gratings," Opt. Lett. 36, 2104-2106 (2011).

22. Y. C. Tan, W. B. Ji, V. Mamidala, K. K. Chow, and S. C. Tjin, "Carbonnanotube-deposited long period fiber grating for continuous refractive index sensor applications," Sens. Actuators B Chem. 196, 260-264 (2014).

23. Y. J. Rao, "In-fibre Bragg grating sensors," Meas. Sci. Technol. 8, 355 (1997).

24. R. Ismaeel, T. Lee, M. Ding, M. Belal, and G. Brambilla, "Optical microfiber passive components," Laser Photonics Rev. 7, 350-384 (2013).

25. A. Zhang, Y. Wu, B. Yao, and Y. Gong, "Optimization study on graphenecoated microfiber Bragg grating structures for ammonia gas sensing," Photonic Sensors 5, 84-90 (2015).

26. E. Cibula, and D. Donlagic, "In-Line Fabry-Pérot Refractive Index Sensor," IEEE Photon. Technol. Lett. 23, 1609-1611 (2011).

27. K. Kashiwagi, and S. Yamashita, "Deposition of carbon nanotubes around microfiber via evanascent light," Opt. Express 17, 18364-18370 (2009).

28. N. D. Rees, S. W. James, R. P. Tatam, and G. J. Ashwell, "Optical fiber long-period gratings with Langmuir-Blodgett thin-film overlays," Opt. Lett. 27, 686-688 (2002).

29. P. Wang, G. Brambilla, M. Ding, Y. Semenova, Q. Wu, and G. Farrell, "High-sensitivity, evanescent field refractometric sensor based on a tapered, multimode fiber interference," Opt. Lett. 36, 2233-2235 (2011).

30. H. J. Patrick, A. D. Kersey, and F. Bucholtz, "Analysis of the response of long period fiber gratings to external index of refraction," J. Lightwave Technol. 16, 1606-1612 (1998). 